\title{
A Novel Method of Beamforming to Improve the Space Diversity
}

\author{
Marco Antonio Acevedo Mosqueda, Emmanuel Martínez Zavala, \\ María Elena Acevedo Mosqueda, and Oleksiy Pogrebnyak
}

\begin{abstract}
At present, the systems of mobile communications demand major capacity in the services, therefore optimum space diversity is needed. In this paper, we propose a new method of beam-forming to improve the space diversity and achieve frequency reuse. Our proposal consists in solving a system of linear equations from an 8-elements linear antenna array that increases the size of the side lobes. This method is compared with the algorithm of Dolph-Chebyshev. Our method shows competitive results in increasing the space diversity.
\end{abstract}

Index Terms-Beamforming, Space diversity, Linear antenna array, Dolph-Chebyshev.

\section{INTRODUCTION}

I $\mathrm{N}$ RECENT DECADES, the interest in the antenna array has grown significantly due to it plays an important role in mobile communications systems. Now these systems demand more services due to in the constant increase in the number of users, and as a possible solution to this problem several studies have focused on a new mechanism known as division multiple access space (Space Division Multiple Access, SDMA) [1-7] which provides the ability to reuse resources (frequency bands, slots time, codes or any combination) simultaneously within the same service area. The goal of this paper is to propose a method to optimize the space diversity of a linear antenna array. A new technique of solving linear equations of a linear array of 8 elements to increase the magnitude of the side lobes and improve space diversity is proposed. This method is compared with the array factor of Dolph-Chebyshev [8] which is used to improve space diversity and it is considered important because it has the largest amplitude of side lobes around the main lobe side.

In Section II the method for designing a weighted and symmetrical array factor (AF) [9] is described. The array has a total of elements equal to 8 and an equidistant separation between the elements of $d=\lambda / 2$. Section III will show the algorithm for calculating the factor of Dolph-Chebyshev array [8], with a Chebyshev polynomial order $m=8$ and a side lobe level $R=15 \mathrm{~dB}$ [10]

The radiation pattern of Section II is used for a first comparison with the pattern of Dolph-Chebyshev radiation in Section III.

Manuscript received April 17, 2012. Manuscript accepted November 20, 2012

M.A. Acevedo, E. Martinez, and M.E. Acevedo are with the Department of Telecommunications Section of Postgraduate Studies and Research, School of Mechanical and Electrical Engineering Zacatenco, DF, Mexico (email: macevedo@ipn.mx,emz06@hotmail.com, eacevedo@ipn.mx).

O. Pogrebnyak is with the Center for Computing Research, National Polytechnic Institute (CIC-IPN), DF, México (e-mail: olek@cic.ipn.mx).
The method to increase secondary lobes is described in Section IV and finally, Section V presents different simulations to obtain a radiation pattern by increasing the magnitude of side lobes, which is comparable to the radiation pattern of Dolph-Chebyshev which has a polynomial order of $m=8$ and a maximum ratio of $4 \mathrm{~dB}$ side lobes.

\section{ARRAY FACTOR FOR LINEAR WEIGHTED UNIFORM SYMMETRICAL ARRAYS}

An antenna array is a set of simple antennas which are connected under certain conditions, and they are usually equal and oriented in the same direction. They are arranged in a specific physical layout and relatively close with respect to each other. The combination of all these parameters provides a unique radiation pattern with desirable features such as width of the main lobe, the location of zero and maximum angles, among others [11].

Figure 1 shows the linear weighted uniform symmetrical array used in this work.

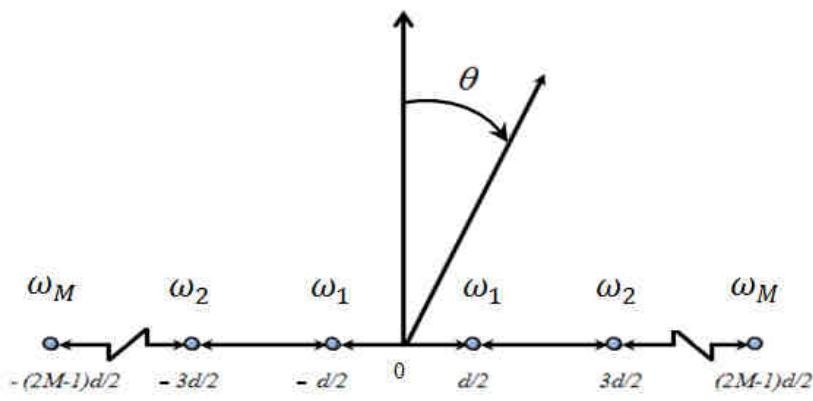

Fig 1. Linear weighted uniform symmetrical array.

In Figure $1, d$ is the distance between adjacent elements, $\theta$ is the angle of measurement and $w_{M}$ are the respective weights for each element of the linear array.

The array factor is obtained by adding the result of the weighing of each element such that:

$$
\begin{array}{r}
F A=\omega_{M} e^{-j \frac{(2 M-1)}{2} k d \sin \theta}+\ldots+\omega_{1} e^{-j \frac{1}{2} k d \sin \theta}+ \\
\omega_{1} e^{j \frac{1}{2} k d \sin \theta}+\ldots+\omega_{M} e^{j \frac{(2 M-1)}{2} k d \sin \theta}
\end{array},
$$

where $k=\frac{2 \pi}{\lambda}$. We can express the above formula in terms of the cosine function using Euler's identity so that:

$$
F A=\sum_{n=1}^{8} \omega_{n} \cos ((2 n-1) u)
$$


where $u=k d \sin \theta$, note that the array factor is maximum if the angle $\theta=0$.

In this paper we use the following array factor for a number of elements equal to 8 with an equidistant distance between elements of $d=\lambda / 2$.

$$
\begin{aligned}
F A= & \omega_{1} e^{-j \frac{(2 \cdot 4-1)}{2} k d \sin \theta}+\omega_{2} e^{-j \frac{(2 \cdot 3-1)}{2} k d \sin \theta}+ \\
& \omega_{3} e^{-j \frac{(2 \cdot 2-1)}{2} k d \sin \theta}+\omega_{4} e^{-j \frac{1}{2} k d \sin \theta}+ \\
& \omega_{5} e^{j \frac{1}{2} k d \sin \theta}+\omega_{6} e^{j \frac{(2 \cdot 2-1)}{2} k d \sin \theta}+ \\
& \omega_{7} e^{j \frac{(2 \cdot 3-1)}{2} k d \sin \theta}+\omega_{8} e^{j \frac{(2 \cdot 4-1)}{2} k d \sin \theta}
\end{aligned}
$$

Figure 2 shows the radiation pattern of the array factor used in this article.

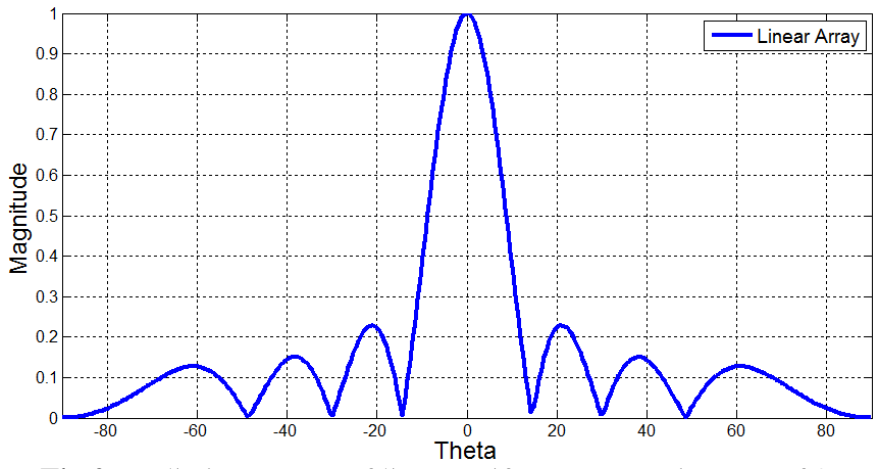

Fig 2. Radiation pattern of linear uniform symmetric array of 8 elements and equidistant distance of $d=\lambda / 2$.

Null or zero angles of the array factor is obtained when:

$\theta_{\text {nulls }}=\sin ^{-1}\left(\frac{1}{k d}\left( \pm \frac{2 n \pi}{N}-\delta\right)\right), \quad n=1,2,3, \ldots$

where $N$ is element array, $\delta$ is the difference of the electrical phase between two adjacent elements. For real angles $\sin \theta$ is less than or equal to one, therefore, the argument of the equation must be less than or equal to one, this means that only a finite set of values satisfy the equality.

The angles of the side lobes are the greatest when:

$\theta_{s}=\sin ^{-1}\left(\frac{1}{k d}\left( \pm \frac{(2 n+1) \pi}{N}-\delta\right)\right) \quad n=1,2,3, \ldots$

The width of the main beam is defined as the angular aperture of the main lobe measured at a given constant power level and it is obtained when:

$$
\theta_{ \pm}=\sin ^{-1}\left(\frac{1}{k d}\left(\frac{ \pm 2.782}{N}-\delta\right)\right)
$$

where $\theta_{+}$and $\theta_{-}$are half-power points and they are normalized to a $F A=0.707$. The bandwidth of medium power is determined as follows:

$$
H P B W=\left|\theta_{+}-\theta_{-}\right|
$$

For an 8-elements array factor, the positive and negative angles where maximums and zeros appear are shown in Table I.

\section{TABle I. Positive AND NeGATIVE ANGLES FOR MAXIMUM AND NULL.}

\begin{tabular}{cc}
\hline Maximum & Nulls \\
\hline $\pm 22.02^{\circ}$ & $\pm 14.47^{\circ}$ \\
$\pm 38.68^{\circ}$ & $\pm 30.00^{\circ}$ \\
$\pm 61.04^{\circ}$ & $\pm 48.59^{\circ}$ \\
$\pm 119.8^{\circ}$ & $\pm 90.00^{\circ}$ \\
\hline
\end{tabular}

\section{DOLPH-CHEBYSHEV ARRAY FACTOR}

The method Dolph-Chebyshev Array was initially investigated by Dolph and it is based on the studies by Chebyshev.

Dolph in 1946 proposed a method to calculate the array factor; this method is based on the approximation to the structure of the linear array by means of the Chebyshev polynomials of order $m$ [9].

\section{1) The Chebyshev polynomials (ChP)}

The ChP of order $m$ are defined as:

$$
T_{m}(z)=\left\{\begin{array}{lr}
(-1)^{m} \cosh (m \cdot \operatorname{arccosh}|z|) & z \leq 1 \\
\cos (m \cdot \arccos (z)) & -1 \leq z \leq 1 \\
\cosh (m \cdot \operatorname{arccosh}(z)) & z \geq 1
\end{array}\right.
$$

Chebyshev polynomials can also be generated using the following recurrent formula:

$$
T_{m}(z)=2 z T_{m-1}(z)-T_{m-2}(z) .
$$

We find the first $\mathrm{ChP}$ by evaluating equation (9), and the first eight polynomials are:

$$
\begin{aligned}
& t_{0}(z)=1 \\
& t_{1}(z)=z \\
& t_{2}(z)=2 z^{2}-1 \\
& t_{3}(z)=4 z^{3}-3 z \\
& t_{4}(z)=8 z^{4}-8 z^{2}+1 \\
& t_{5}(z)=16 z^{5}-20 z^{3}+5 z \\
& t_{6}(z)=32 z^{6}-48 z^{4}+18 z^{2}-1 \\
& t_{7}(z)=64 z^{7}-112 z^{5}+56 z^{3}-7 z
\end{aligned}
$$

It can be observed that if the module of $z$ is greater than or equal to 1 then the $\mathrm{ChP}$ are directly related to the cosine function.

\section{2) Properties of Chebyshev polynomials}

We must take into account some of the properties of Chebyshev polynomials: 
a) All polynomials of any $m$ order will pass through the point $(1,1)$.

b) Within the range $-1 \leq z \leq 1$, the polynomials have values in $[-1,1]$.

c) All null values occur within the range $-1 \leq z \leq 1$.

d) The maximum and minimum values within the range $z$ $\in[-1,1]$ have values +1 and -1 respectively.

e) The higher the order of the polynomial, the steeper the slope of $|z| \geq 1$

The zeros of Chebyshev polynomials are calculated as follows:

$$
\cos \left[\frac{(2 m-1) \cdot \pi}{2 N}\right], \quad m=0,1, \ldots, N-1
$$

\section{3) The designing of the Dolph-Chebyshev Array Factor}

The approximation of the linear array of antennas through the ChP is accomplished by the proposal of the number of elements in the array, in this case we proposed $N=8$.

$$
m=N-1
$$

where $m$ is the order of Chebyshev polynomial. Chebyshev polynomials are identical to equation (2), if we write them as follows:

$$
T_{N-1}(z)=\sum_{n=1}^{M} a_{n} \cos [(2 n-1) u]
$$

where $=\left(\frac{\pi \cdot d}{\lambda}\right) \cos \theta, a_{n}=$ Chebyshev coefficients.

The level of side lobes in $\mathrm{dB}$ and the rectangular shape can be obtained by:

$$
R_{o}=\frac{1}{\left|F A_{l s}\right|}, \quad R=20 \log _{10}\left(R_{o}\right), \quad R_{o}=10^{R / 20},
$$

where $F A_{l s}$ is array factor of side lobes, $R_{o}$ is the level of the main lobe and $R$ is the level of the side lobes with respect to the main lobe.

Figure 3 shows the radiation pattern generated by a DolphChebyshev-type amplitude distribution with a total number of elements $N=8$ and a ratio of lobes of $R=15 \mathrm{~dB}$.

A comparison between the radiation patterns from the original array factor and the Dolph-Chebyshev method is shown Figure 4.

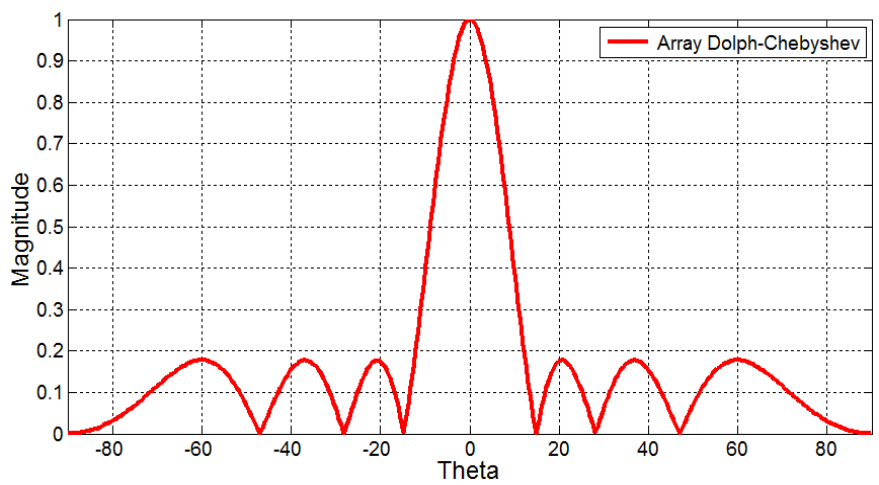

Fig 3. Radiation Pattern of 8-element Dolph-Chebyshev and a ratio of lobes of $15 \mathrm{~dB}$.

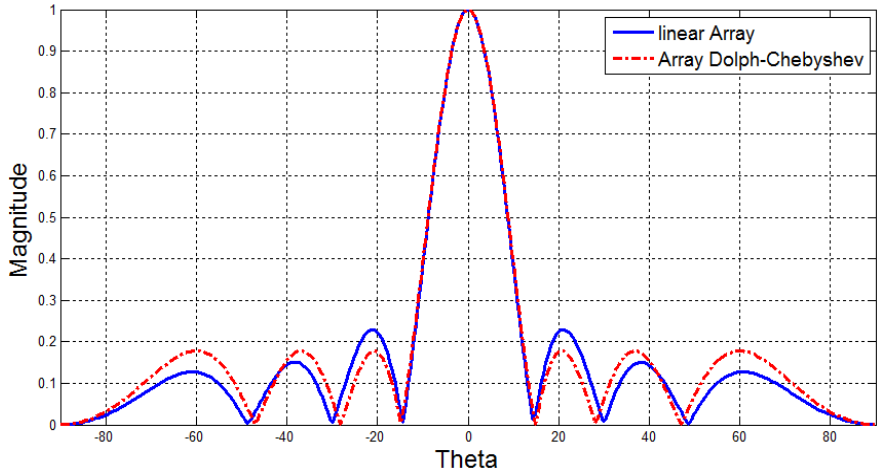

Fig. 4. Radiation Patterns from Linear Array and DolphChebyshev.

In Figure 4 we can observe that the width of the main lobe is the same in both radiation patterns. The amplitude of the first side lobe of the radiation pattern from the linear array is greater than the amplitude of the first side lobe of the radiation pattern from Dolph-Chebyshev method.

The level of the amplitude of the second and third secondary lobes of the radiation pattern from DolphChebyshev are greater than the second and third side lobes of the radiation pattern from linear array. The first null in both radiation patterns is located at the same angle, but the second and third nulls from Dolph-Chebyshev are shifted with respect to those from the linear array.

This comparison suggests that there is not a significant difference between the two radiation patterns.

\section{METHOD FOR INCREASING SIDE LOBES}

For increasing the side lobes the weighing of the antenna array has to be chosen so that a null value will be placed in calculated directions and a maximum value will be placed in the angles of the side lobes and leaving the peak of the main lobe in the direction of interest [10].

For a set of 8 elements the vector of the array is given by:

$$
\begin{array}{r}
\bar{a}(\theta)=\left[e^{-j \frac{7}{2} k d \sin \theta} e^{-j \frac{5}{2} k d \sin \theta} e^{-j \frac{3}{2} k d \sin \theta} e^{-j \frac{1}{2} k d \sin \theta}\right. \\
\left.e^{j \frac{1}{2} k d \sin \theta} e^{j \frac{3}{2} k d \sin \theta} e^{j \frac{5}{2} k d \sin \theta} e^{j \frac{7}{2} k d \sin \theta}\right]^{T}
\end{array}
$$

The weighting vector is described as follows:

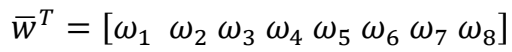

Therefore the total array is the sum of the vector array plus the weighting vector obtained:

$$
\begin{gathered}
S=\bar{w}^{T} \cdot \bar{a}=\omega_{1} e^{-j \frac{7}{2} k d \sin \theta}+\omega_{2} e^{-j \frac{5}{2} k d \sin \theta}+\omega_{3} e^{-j \frac{3}{2} k d \sin \theta}+\omega_{4} e^{-j \frac{1}{2} k d \sin \theta} \\
+\omega_{5} e^{j \frac{1}{2} k d \sin \theta}+\omega_{6} e^{j \frac{3}{2} k d \sin \theta}+\omega_{7} e^{j \frac{5}{2} k d \sin \theta}+\omega_{8} e^{j \frac{7}{2} k d \sin \theta}
\end{gathered}
$$

Since there are 8 variables we have 8 conditions to satisfy:

$$
\begin{gathered}
S_{1}=\omega_{1} e^{-j \frac{7}{2} k d \sin \theta_{1}}+\omega_{1} e^{-j \frac{5}{2} k d \sin \theta_{1}}+\omega_{1} e^{-j \frac{3}{2} k d \sin \theta_{1}}+\omega_{1} e^{-j \frac{1}{2} k d \sin \theta_{1}} \\
+\omega_{1} e^{j \frac{1}{2} k d \sin \theta_{1}}+\omega_{1} e^{j \frac{3}{2} k d \sin \theta_{1}}+\omega_{1} e^{j \frac{5}{2} k d \sin \theta_{1}}+\omega_{1} e^{j \frac{7}{2} k d \sin \theta_{1}}=\boldsymbol{c} \mathbf{1}
\end{gathered}
$$




$$
\begin{aligned}
& S_{2}=\omega_{1} e^{-j \frac{7}{2} k d \sin \theta_{2}}+\omega_{2} e^{-j \frac{5}{2} k d \sin \theta_{2}}+\omega_{3} e^{-j \frac{3}{2} k d \sin \theta_{2}}+\omega_{4} e^{-j \frac{1}{2} k d \sin \theta_{2}} \\
& +\omega_{5} e^{j \frac{1}{2} k d \sin \theta_{2}}+\omega_{6} e^{j \frac{3}{2} k d \sin \theta_{2}}+\omega_{7} e^{j \frac{5}{2} k d \sin \theta_{2}}+\omega_{8} e^{j \frac{7}{2} k d \sin \theta_{2}}=\mathbf{c 2} \\
& S_{3}=\omega_{1} e^{-j \frac{7}{2} k d \sin \theta_{3}}+\omega_{2} e^{-j \frac{5}{2} k d \sin \theta_{3}}+\omega_{3} e^{-j \frac{3}{2} k d \sin \theta_{3}}+\omega_{4} e^{-j \frac{1}{2} k d \sin \theta_{3}} \\
& +\omega_{5} e^{j \frac{1}{2} k d \sin \theta_{3}}+\omega_{6} e^{j \frac{3}{2} k d \sin \theta_{3}}+\omega_{7} e^{j \frac{5}{2} k d \sin \theta_{3}}+\omega_{8} e^{j \frac{7}{2} k d \sin \theta_{3}}=\mathbf{c 3} \\
& S_{4}=\omega_{1} e^{-j \frac{7}{2} k d \sin \theta_{4}}+\omega_{2} e^{-j \frac{5}{2} k d \sin \theta_{4}}+\omega_{3} e^{-j \frac{3}{2} k d \sin \theta_{4}}+\omega_{4} e^{-j \frac{1}{2} k d \sin \theta_{4}} \\
& +\omega_{5} e^{j \frac{1}{2} k d \sin \theta_{4}}+\omega_{6} e^{j \frac{3}{2} k d \sin \theta_{4}}+\omega_{7} e^{j \frac{5}{2} k d \sin \theta_{4}}+\omega_{8} e^{j \frac{7}{2} k d \sin \theta_{4}}=\boldsymbol{c} \mathbf{4} \\
& S_{5}=\omega_{1} e^{-j \frac{7}{2} k d \sin \theta_{5}}+\omega_{2} e^{-j \frac{5}{2} k d \sin \theta_{5}}+\omega_{3} e^{-j \frac{3}{2} k d \sin \theta_{5}}+\omega_{4} e^{-j \frac{1}{2} k d \sin \theta_{5}} \\
& +\omega_{5} e^{j \frac{1}{2} k d \sin \theta_{5}}+\omega_{6} e^{j \frac{3}{2} k d \sin \theta_{5}}+\omega_{7} e^{j \frac{5}{2} k d \sin \theta_{5}}+\omega_{8} e^{j \frac{7}{2} k d \sin \theta_{5}}=\mathbf{c 5} \\
& S_{6}=\omega_{1} e^{-j \frac{7}{2} k d \sin \theta_{6}}+\omega_{2} e^{-j \frac{5}{2} k d \sin \theta_{6}}+\omega_{3} e^{-j \frac{3}{2} k d \sin \theta_{6}}+\omega_{4} e^{-j \frac{1}{2} k d \sin \theta_{6}} \\
& +\omega_{5} e^{j \frac{1}{2} k d \sin \theta_{6}}+\omega_{6} e^{j \frac{3}{2} k d \sin \theta_{6}}+\omega_{7} e^{j \frac{5}{2} k d \sin \theta_{6}}+\omega_{8} e^{j \frac{7}{2} k d \sin \theta_{6}}=\mathbf{c 6} \\
& S_{7}=\omega_{1} e^{-j \frac{7}{2} k d \sin \theta_{7}}+\omega_{2} e^{-j \frac{5}{2} k d \sin \theta_{7}}+\omega_{3} e^{-j \frac{3}{2} k d \sin \theta_{7}}+\omega_{4} e^{-j \frac{1}{2} k d \sin \theta_{7}} \\
& +\omega_{5} e^{j \frac{1}{2} k d \sin \theta_{7}}+\omega_{6} e^{j \frac{3}{2} k d \sin \theta_{7}}+\omega_{7} e^{j \frac{5}{2} k d \sin \theta_{7}}+\omega_{8} e^{j \frac{7}{2} k d \sin \theta_{7}}=\boldsymbol{c 7} \\
& S_{8}=\omega_{1} e^{-j \frac{7}{2} k d \sin \theta_{8}}+\omega_{2} e^{-j \frac{5}{2} k d \sin \theta_{8}}+\omega_{3} e^{-j \frac{3}{2} k d \sin \theta_{8}}+\omega_{4} e^{-j \frac{1}{2} k d \sin \theta_{8}} \\
& +\omega_{5} e^{j \frac{1}{2} k d \sin \theta_{8}}+\omega_{6} e^{j \frac{3}{2} k d \sin \theta_{8}}+\omega_{7} e^{j \frac{5}{2} k d \sin \theta_{8}}+\omega_{8} e^{j \frac{7}{2} k d \sin \theta_{8}}=\boldsymbol{c 8}
\end{aligned}
$$

where $c 1, \cdots, c 8$ are constant. We have a system of equations where each equation is equal to the magnitude of the desired level of secondary lobes which is trying to keep the width of the main lobe in the half-power points. Solving this system of equations the weights can be obtained for each condition.

\section{Simulation}

In this section, we describe the process for increasing the secondary lobes in a linear array of 8-element antenna with an equidistant distance between elements of $d=\lambda / 2$.

\section{Case I}

Increase the first positive side lobe at magnitude of 0.7 . For this we will use a combination of maximum and zero angles which are shown in Table II.

TABLE II. ANGLE COMBINATION FOR INCREASING THE FIRST POSITIVE SIDE LOBE

\begin{tabular}{|c|c|c|c|}
\hline$\theta_{1}=-90^{*} r$ & $\theta_{2}=-48.59^{*} r$ & $\theta_{3}=-30^{*} r$ & $\theta_{4}=-14.47^{*} r$ \\
\hline$\theta_{5}=14.47^{*} r$ & $\theta_{6}=22.02^{*} r$ & $\theta_{7}=48.59^{*} r$ & $\theta_{8}=90^{*} r$ \\
\hline
\end{tabular}

In Table II, $r=\pi d 180$ and the angle $\theta_{6}$ represents the direction in which we require to increase the side lobe so that the equation will be matched to a desired maximum magnitude value. The angles $\theta_{4}$ and $\theta_{5}$ will not change because they are required to keep the width of the main lobe, while the remaining angles will be null for other directions.

These angles are replaced in the following equation system as follows:

$$
\begin{gathered}
S_{1}=\omega_{1} e^{-j \frac{7}{2} k d \sin \theta_{1}}+\omega_{1} e^{-j \frac{5}{2} k d \sin \theta_{1}}+\omega_{1} e^{-j \frac{3}{2} k d \sin \theta_{1}}+\omega_{1} e^{-j \frac{1}{2} k d \sin \theta_{1}} \\
+\omega_{1} e^{j \frac{1}{2} k d \sin \theta_{1}}+\omega_{1} e^{j \frac{3}{2} k d \sin \theta_{1}}+\omega_{1} e^{j \frac{5}{2} k d \sin \theta_{1}}+\omega_{1} e^{j \frac{7}{2} k d \sin \theta_{1}}=\mathbf{0} \\
S_{2}=\omega_{1} e^{-j \frac{7}{2} k d \sin \theta_{2}}+\omega_{2} e^{-j \frac{5}{2} k d \sin \theta_{2}}+\omega_{3} e^{-j \frac{3}{2} k d \sin \theta_{2}}+\omega_{4} e^{-j \frac{1}{2} k d \sin \theta_{2}}
\end{gathered}
$$

$$
\begin{aligned}
& +\omega_{5} e^{j \frac{1}{2} k d \sin \theta_{2}}+\omega_{6} e^{j \frac{3}{2} k d \sin \theta_{2}}+\omega_{7} e^{j \frac{5}{2} k d \sin \theta_{2}}+\omega_{8} e^{j \frac{7}{2} k d \sin \theta_{2}}=\mathbf{0} \\
& S_{3}=\omega_{1} e^{-j \frac{7}{2} k d \sin \theta_{3}}+\omega_{2} e^{-j \frac{5}{2} k d \sin \theta_{3}}+\omega_{3} e^{-j \frac{3}{2} k d \sin \theta_{3}}+\omega_{4} e^{-j \frac{1}{2} k d \sin \theta_{3}} \\
& +\omega_{5} e^{j \frac{1}{2} k d \sin \theta_{3}}+\omega_{6} e^{j \frac{3}{2} k d \sin \theta_{3}}+\omega_{7} e^{j \frac{5}{2} k d \sin \theta_{3}}+\omega_{8} e^{j \frac{7}{2} k d \sin \theta_{3}}=\mathbf{0} \\
& S_{4}=\omega_{1} e^{-j \frac{7}{2} k d \sin \theta_{4}}+\omega_{2} e^{-j \frac{5}{2} k d \sin \theta_{4}}+\omega_{3} e^{-j \frac{3}{2} k d \sin \theta_{4}}+\omega_{4} e^{-j \frac{1}{2} k d \sin \theta_{4}} \\
& +\omega_{5} e^{j \frac{1}{2} k d \sin \theta_{4}}+\omega_{6} e^{j \frac{3}{2} k d \sin \theta_{4}}+\omega_{7} e^{j \frac{5}{2} k d \sin \theta_{4}}+\omega_{8} e^{j \frac{7}{2} k d \sin \theta_{4}}=\mathbf{0 . 7} \\
& S_{5}=\omega_{1} e^{-j \frac{7}{2} k d \sin \theta_{5}}+\omega_{2} e^{-j \frac{5}{2} k d \sin \theta_{5}}+\omega_{3} e^{-j \frac{3}{2} k d \sin \theta_{5}}+\omega_{4} e^{-j \frac{1}{2} k d \sin \theta_{5}} \\
& +\omega_{5} e^{\frac{1}{2} k d \sin \theta_{5}}+\omega_{6} e^{j \frac{3}{2} k d \sin \theta_{5}}+\omega_{7} e^{j \frac{5}{2} k d \sin \theta_{5}}+\omega_{8} e^{j \frac{7}{2} k d \sin \theta_{5}}=\mathbf{0 . 7} \\
& S_{6}=\omega_{1} e^{-j \frac{7}{2} k d \sin \theta_{6}}+\omega_{2} e^{-j \frac{5}{2} k d \sin \theta_{6}}+\omega_{3} e^{-j \frac{3}{2} k d \sin \theta_{6}}+\omega_{4} e^{-j \frac{1}{2} k d \sin \theta_{6}} \\
& +\omega_{5} e^{j \frac{1}{2} k d \sin \theta_{6}}+\omega_{6} e^{j \frac{3}{2} k d \sin \theta_{6}}+\omega_{7} e^{j \frac{5}{2} k d \sin \theta_{6}}+\omega_{8} e^{j \frac{7}{2} k d \sin \theta_{6}}=\mathbf{0 . 7} \\
& S_{7}=\omega_{1} e^{-j \frac{7}{2} k d \sin \theta_{7}}+\omega_{2} e^{-j \frac{5}{2} k d \sin \theta_{7}}+\omega_{3} e^{-j \frac{3}{2} k d \sin \theta_{7}}+\omega_{4} e^{-j \frac{1}{2} k d \sin \theta_{7}} \\
& +\omega_{5} e^{j \frac{1}{2} k d \sin \theta_{7}}+\omega_{6} e^{j \frac{3}{2} k d \sin \theta_{7}}+\omega_{7} e^{j \frac{5}{2} k d \sin \theta_{7}}+\omega_{8} e^{j \frac{7}{2} k d \sin \theta_{7}}=\mathbf{0} \\
& S_{8}=\omega_{1} e^{-j \frac{7}{2} k d \sin \theta_{8}}+\omega_{2} e^{-j \frac{5}{2} k d \sin \theta_{8}}+\omega_{3} e^{-j \frac{3}{2} k d \sin \theta_{8}}+\omega_{4} e^{-j \frac{1}{2} k d \sin \theta_{8}} \\
& +\omega_{5} e^{j \frac{1}{2} k d \sin \theta_{8}}+\omega_{6} e^{j \frac{3}{2} k d \sin \theta_{8}}+\omega_{7} e^{j \frac{5}{2} k d \sin \theta_{8}}+\omega_{8} e^{j \frac{7}{2} k d \sin \theta_{8}}=\mathbf{0}
\end{aligned}
$$

The result from this equation system will be 8 weights that will satisfy each condition. The obtained weights are substituted in the array factor in the formula (17).

This process will be held in every case to obtain the radiation pattern. Figure 5 shows the radiation pattern obtained from the first case:

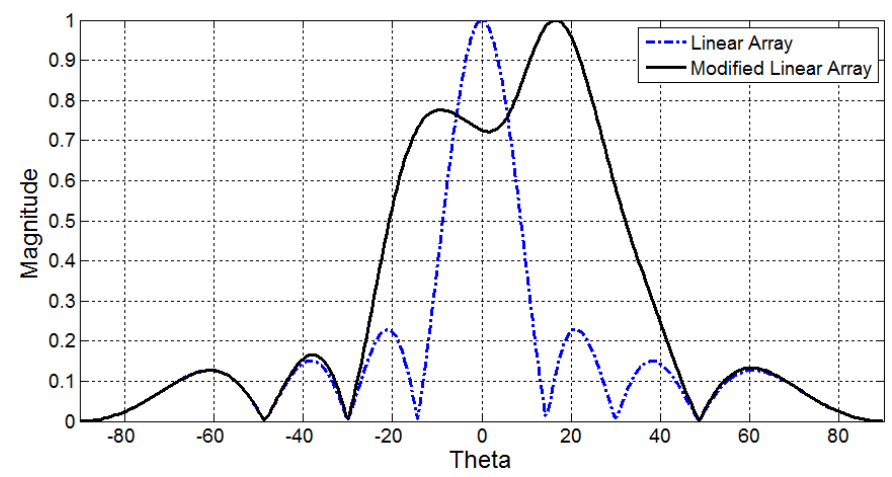

Fig 5. Radiation pattern of the first linear array by increasing the positive side lobe.

In Figure 5, we can observe the increase of the first positive side lobe, and due to there is not a null between the two maximum angles then a continuity between the main lobe and side lobe of the first maximum is shaped; therefore, a spread of the main lobe is generated by the joint of the highs from the two lobes.

We can observe that a null appears at the first negative angle which is at $-30^{\circ}$, the second angle is at $-48.59^{\circ}$ and the third is at $90^{\circ}$. The first null at a positive angle is found at $48.59^{\circ}$ and the second angle is at $90^{\circ}$ as it is indicated in the equations.

Table III shows the combinations of angles which generate maximum and null values. 
TABLE III. COMBINATION OF ANGLES FOR INCREASING THE SIDE LOBES.

\begin{tabular}{|c|c|c|c|}
\hline \multicolumn{4}{|c|}{ Case II } \\
\hline$\theta_{1}=-90^{*} r$ & $\theta_{2}=-48.59 * r$ & $\theta_{3}=-30^{*} r$ & $\theta_{4}=-14.47^{*} r$ \\
\hline 0 & 0 & 0 & 0.7 \\
\hline$\theta_{5}=14.47^{*} r$ & $\theta_{6}=30^{*} r$ & $\theta_{7}=38.68^{*} r$ & $\theta_{8}=90^{*} \mathrm{r}$ \\
\hline 0.7 & 0 & 0.7 & 0 \\
\hline \multicolumn{4}{|c|}{ Case III } \\
\hline$\theta_{1}=-90^{*} r$ & $\theta_{2}=-48.59{ }^{*} r$ & $\theta_{3}=-30^{*} r$ & $\theta_{4}=-14.47^{*} r$ \\
\hline 0 & 0 & 0 & 0.7 \\
\hline$\theta_{5}=14.47^{*} r$ & $\theta_{6}=30 * r$ & $\theta_{7}=48.59^{*} r$ & $\theta_{8}=61.04^{*} r$ \\
\hline 0.7 & 0 & 0 & 0.7 \\
\hline \multicolumn{4}{|c|}{ Case IV } \\
\hline$\theta_{1}=-61.04^{*} r$ & $\theta_{2}=-48.59{ }^{*} r$ & $\theta_{3}=-30 * r$ & $\theta_{4}=-14.47^{*} r$ \\
\hline 0.7 & 0 & 0 & 0.7 \\
\hline$\theta_{5}=14.47^{*} r$ & $\theta_{6}=30 * r$ & $\theta_{7}=48.59^{*} r$ & $\theta_{8}=61.04^{*} r$ \\
\hline 0.7 & 0 & 0 & 0.7 \\
\hline \multicolumn{4}{|c|}{ Case $V$} \\
\hline$\theta_{1}=-61.04^{*} r$ & $\theta_{2}=-48.59 * r$ & $\theta_{3}=-22.02{ }^{*} r$ & $\theta_{4}=-14.47^{*} r$ \\
\hline 0.7 & 0 & 0.7 & 0.7 \\
\hline$\theta_{5}=14.47^{*} r$ & $\theta_{6}=22.02^{*} r$ & $\theta_{7}=48.59^{*} r$ & $\theta_{B}=61.04^{*} r$ \\
\hline 0.7 & 0.7 & 0 & 0.7 \\
\hline
\end{tabular}

\section{Case II}

We increase the second positive side lobe with a magnitude of 0.7 . Once the equation system has been solved and taking into account the obtained weight in case I, then the weights will be multiplied as follows:

$$
\begin{aligned}
\omega^{T}= & {\left[\omega_{1}^{I} \cdot \omega_{1}^{I I}=\omega_{1} ; \omega_{2}^{I} \cdot \omega_{2}^{I I}=\omega_{2} ;\right.} \\
& \omega_{3}^{I} \cdot \omega_{3}^{I I}=\omega_{3} ; \omega_{4}^{I} \cdot \omega_{4}^{I I}=\omega_{4} ; \\
& \omega_{5}^{I} \cdot \omega_{5}^{I I}=\omega_{5} ; \omega_{6}^{I} \cdot \omega_{6}^{I I}=\omega_{6} ; \\
& \left.\omega_{7}^{I} \cdot \omega_{7}^{I I}=\omega_{7} ; \omega_{8}^{I} \cdot \omega_{8}^{I I}=\omega_{8} ;\right]
\end{aligned}
$$

These new weights are replaced in the linear array factor and the result is the radiation pattern that is shown in Figure 6. Equation (21) will be applied in the same way in each and every of the later cases.

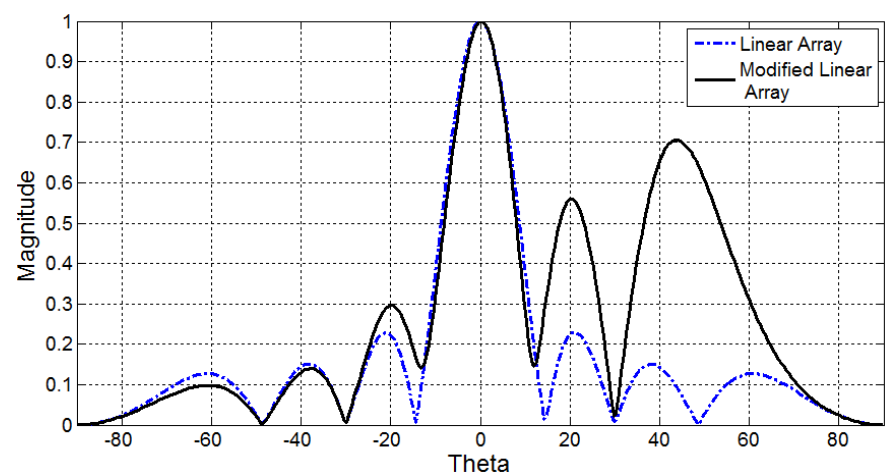

Fig 6. Radiation pattern from the linear array when the positive second side lobe is increased.

In Figure 6 we observe an increase in the positive side-lobe level, as well as the widening of it. We also observed an increase of the first secondary lobe. The angular opening in the half-power points is preserved.

\section{Case III} 0.7 .

We increase the third positive side lobe to an amplitude of

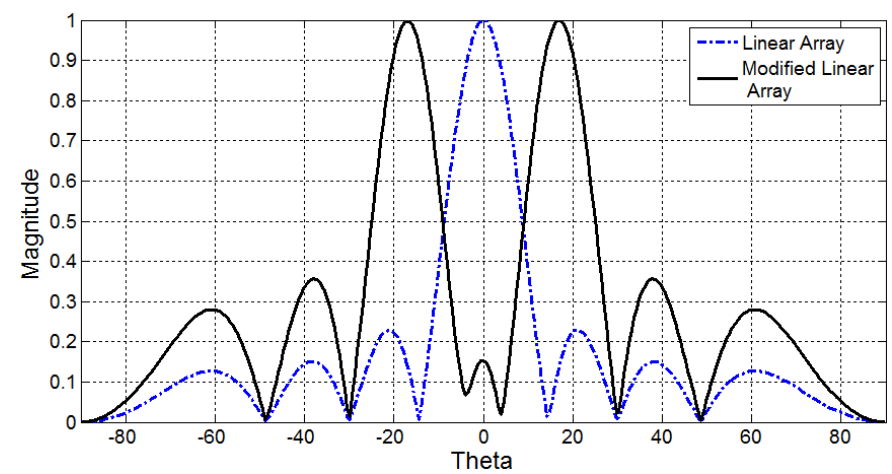

Fig 7. Radiation pattern from linear array when the third positive side lobe is increased.

In Figure 7 we note an increase in amplitude of the positive and negative side lobes, but the direction of interest does not present the desired change of amplitude. We can also observe that there is not a main lobe.

The increase in negative side lobes are not illustrated because the results are symmetrical with respect to the radiation patterns of case I, II, III respectively. In the following cases we only present the most important results to obtain the final radiation pattern.

\section{Case IV}

In this case we use a combination of two maximum angles at different positions. Figure 8 shows the resulting radiation pattern.

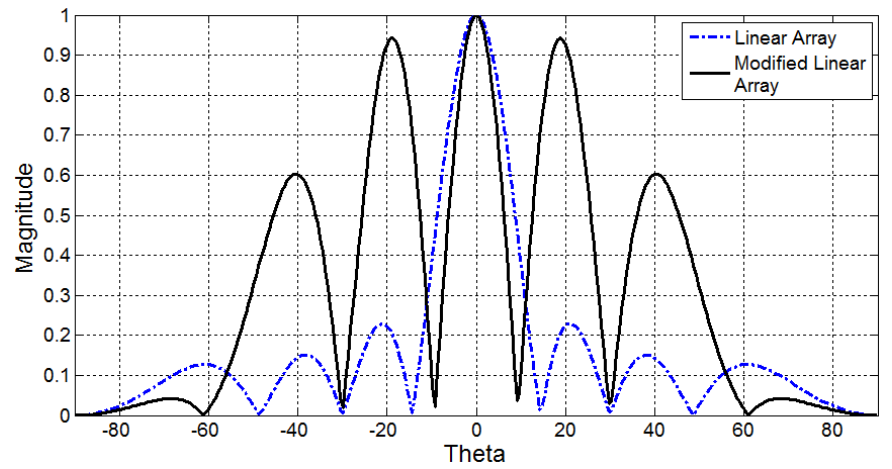

Fig 8. Radiation pattern of linear array by increasing lobes

The width of the main lobe is narrower than the original; this result causes a displacement of null angles. All side lobes had a significant increment in the amplitude except the last two side lobes.

\section{Case V: Comparison with Dolph-Chebyshev}

Finally, we performed the comparison between the resulting radiation pattern from linear array with the radiation pattern from Dolph-Chebyshev method. The DolphChebyshev polynomial will be of order $m=8$ and a maximum ratio between side lobes of $4 \mathrm{~dB}$. Figure 9 shows the 
comparison among the three methods that we discussed in this work: Linear array, Modified linear array and DolphChebyshev. It performs the change in the relative side lobes for comparison.

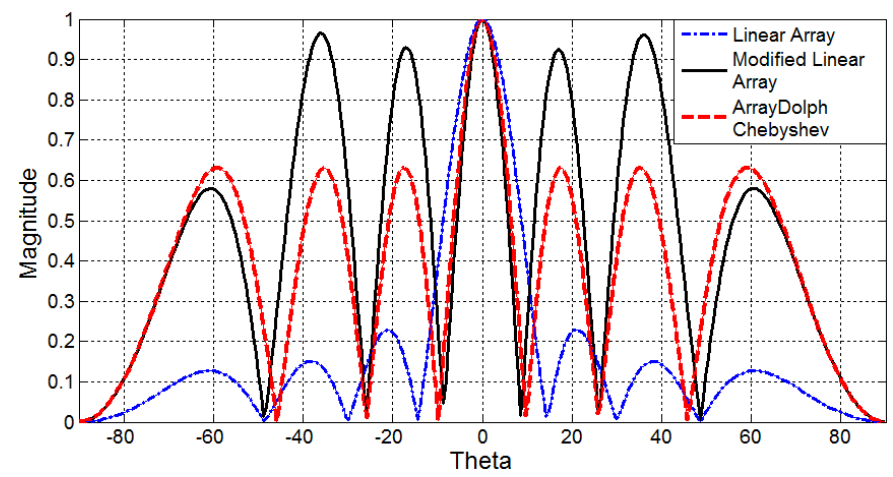

Fig 9. Radiation pattern from original linear array, Modified linear array and Dolph-Chebyshev.

In Figure 9 we can observe that the resulting modified radiation pattern present the highest amplitude in the first two secondary lobes, but this behavior is not uniform because Dolph-Chebyshev amplitude is the highest in the last two side lobes. The amplitude of side lobes from Dolph-Chebyshev is uniform throughout the radiation pattern. In both radiation patterns, Modified linear array and Dolph-Chebyshev, the angular aperture is narrow which causes the null angles to be shifted.

\section{CONCLUSIONS}

The comparison between the method with increased side lobes and the Dolph-Chebyshev method showed similar results, but the amplitude of side lobe of our proposal presented higher level than the amplitude obtained by DolphChebyshev method. The latter method is considered important because it is one that shows the greatest amplitude in side lobes near the main lobe.
Our proposal showed a more directive main lobe and the side lobes have the same level of amplitude as the main lobe. The increase of lobes can be the key to improve the capacity in mobile communication systems, using the frequency reuse concept based on the diversity of space.

Future research aims to achieve uniform increase of all side lobes using adaptive methods.

\section{REFERENCES}

[1] M. Alanis, I. Elshafiey, A. Al-Sanie, "Multi-User Detection for SDMA-OFDM Communication Systems," IEEE, 2011.

[2] A.l. Sulyman, M. Hefnawi, "Capacity-aware linear MMSE detector for OFDM-SDMA Systems", Institution of Engineering and Technology, 2010.

[3] Ch. Santhi Rani, P. V. Subbaiah, K. Chennakesava Reddy, S. Sudha Rani, "LMS and RLS Algorithms for smart antennas in a W-CDMA mobile communication environment, "ARPN Journal of engineering and applied sciences, vol. 4, No. 6, 2009.

[4] S. Bellofiore, J.Foutz, R. Govindarajula,C.A.Balanis, “ Smart antenna system analysis, integration and performance for mobile ad-hoc networks," IEEE, vol. 50, No. 5, 2002.

[5] I. Villordo, I.E. Zaldivar, "An Overview of SDMA in Communications Systems," IEEE, 2006.

[6] M. P. Lotter, P.Rooyen, "Space Division Multiple Access for Cellular CDMA," IEEE, 1998.

[7] G.M. Galvan, M. Botello, "Acceso multiple por division de espacio:una nueva dimension, " ESIME-IPN, 2004.

[8] J.L Ramos, M. J. Martínez, G. A. Vega, M. S. Ruiz, "Software para el cálculo de patrones de radiación de arreglos lineales de antenas," Conferencia de Ingeniería Eléctrica, 2001.

[9] M. A. Acevedo, R.Castañeda, Oleksiy-Pogrebnyak, "Diseño de antenas de ranura resonante para su aplicación en redes WiFi," ESIME-IPN, vol. 13, No.1, 2009.

[10] Gross, Frank, Smart Antennas for Wireless Communication, McGraw-Hill, 2005.

[11] C, A, Balanis, Antenna theory: Analysis and Design, John Wiley and Sons, New York, 1982. 